\section{The effects on speeded classification of implicit and explicit instructions regarding redundant dimensions*}

\author{
GARY L. FELFOLDY and W. R. GARNER \\ Yale University, New Haven, Connecticut 06510
}

Two experiments were run in which speed of sorting decks of stimulus cards was measured. Stimuli were constructed from two dichotomous dimensions, used either alone or perfectly correlated. The lack of evidence for a selective serial processing (SSP) strategy (in which $\mathrm{S}$ sorts by his most preferred dimension whenever the dimensions are correlated) in a similar study by Garner and Felfoldy (1970) was thought to be due to Ss' failure to notice that the dimensions were correlated, and thus that SSP would be effective. Ss in the present experiment therefore received either implicit instructions concerning the existence of the correlated deck (by seeing only decks in which there were just two different stimuli), or explicit instructions that on some trials the two dimensions would be correlated and $\mathrm{S}$ could sort by any means he preferred. When dimensions of size of circle and angle of diameter were of unequal discriminability, implicit instructions produced partial use of the SSP strategy, while explicit instructions produced nearly total use of SSP by all Ss. When the Munsell dimensions of value and chroma were varied in two separate color chips and were equally discriminable-on the average, evidence for a small amount of SSP was found in both the implicit and explicit conditions. With neither pair of dimensions did implicit or explicit instructions regarding the correlated task produce integration of information.

Garner and Felfoldy (1970) examined the effects of redundant dimensions on perceptual facilitation and of orthogonal dimensions on perceptual interference in a speeded classification task. Ss sorted decks of cards into two piles as defined by the dichotomous levels of one of two dimensions as rapidly as possible, while avoiding errors. In one condition only a single dimension varied, the other being held constant, to provide a base-line measure of sorting speed against which to compare the facilitation and interference effects. In a second condition the dimensions varied in a perfectly correlated, or redundant fashion, but $\mathrm{Ss}$ were still asked to sort by only one of the two dimensions. In the third condition the two dimensions varied in an orthogonal, or uncorrelated, manner; S' task was to at tend selectively to the one dimension by which he was instructed to sort. This paradigm was used in each of seven different experiments, the experiments differing only in the specific pair of stimulus dimensions used.

The results were consistent with the hypothesis that pairs of dimensions which produce facilitation (a gain in sorting speed) when they are correlated will also produce interference (a loss in speed) when orthogonal. Furthermore, dimensions which do not produce a redundancy gain

*This research was supported by Grant MH14229 from the National Institute of Mental Health to Yale University. also do not interfere with each other when selective attention to one of the orthogonal stimulus dimensions is required.

\section{Integrality}

These findings, along with the results of several other studies, converged to the concept of dimensional integrality. Dimensions which produced both a redundancy gain and an interference effect a Euclidean metric in direct stimulus scaling (Hyman \& Well, 1968; Shepard, 1964) were termed integral dimensions. Those which produced neither a redundancy gain nor an interference effect in speeded classification, and which led to a city-block metric in direct stimulus scaling (Hyman \& Well. 1968) were termed nonintegral.

Essentially, Garner and Felfoldy (1970) argued that the redundancy gain produced by integral dimensions is the result of increased discriminability produced by the additional correlated dimension. In the truly integral pair of dimensions, the information provided by each dimension probably is not processed separately. Rather, the information from each is integrated, and the discrimination is made on the basis of some redefined, single dimension.

\section{Selective Serial Processing (SSP)}

Information integrating, however, was not the only way in which a redundancy gain could be produced in the Garner and in speeded classification, and which led to
Felfoldy (1970) task. When dimensions are nonintegral and can be processed sequentially, an SSP strategy can lead to such a gain. $S$ merely sorts by the easier dimension whenever he is given the correlated task, regardless of the instructions. He is thus able to produce an average sorting time for the correlated decks which is below that of the single-dimension decks.

There are two conditions which make an SSP sorting strategy effective: Either one dimension is more discriminable than the other for all $S s$ or each $S$ finds one dimension easier than the other, although which dimension is more discriminable varies among Ss. One or the other of these conditions was satisfied in all four of the "nonintegral" experiments of Garner and Felfoldy (1970), yet little or no evidence for SSP was found in any of the four.

\section{Purpose}

The present experiments were designed to examine the conditions affecting the use of the SSP strategy in the card-sorting task. A reasonable explanation of Ss' failure to use this strategy in the earlier experiments is that they failed to recognize that the dimensions were in fact redundant and thus that the SSP strategy would be effective. If this was the case, descriptions of the nature of the correlated task (either implicit or explicit) should increase the use of the SSP strategy.

Any question concerning gains in sorting speed duc to the use of the SSP strategy must simultaneously be concerned with gains due to true integration of information, since improved performance can occur either because dimensions are integral or because they are nonintegral dimensions and the SSP strategy is used. Thus, while the primary purpose of this paper is to determine if the particular experimental procedures used previously led to the failure of Ss to use SSP, its secondary purpose is to determine if these procedures also prevented the integration of information.

\section{GENERAL PROCEDURE}

Two experiments were run, differing only in the specific dimensions which made up the stimuli. Those procedures which were common to both experiments will be described in this section. A description of the dimensions used in each experiment will be included with a discussion of the results.

\section{Subjects and Sorting Task}

In each experiment 24 Ss were used. They were undergraduate and graduate students. paid $\$ 1.75$ to participate as volunteers. Each $S$ participated in a single session lasting from 45 to $65 \mathrm{~min}$. 
Decks of 32 stimulus cards (white, $8.9 \mathrm{~cm}$ high $\times 6.3 \mathrm{~cm}$ wide) were sorted into two piles according to the levels of one of two dimensions. $\mathrm{S}$ and $\mathrm{E}$ faced each other across a table in a 7 -ft-square sound room. $S$ was allowed to examine the first few cards in the deck before each trial. He then sorted the complete deck as fast as possible while avoiding any errors. After each trial $E$ recorded sorting time to the nearest $0.2 \mathrm{sec}$, as well as any errors, and shuffled the next deck of cards to be used. This sorting task was identical to that used by Garner and Felfoldy (1970) except that in the present experiments $S$ was informed of his sorting time and error score after each trial.

\section{Conditions}

Implicit instructions. Twelve different Ss were run in each of two conditions. In one condition Ss were given implicit knowledge of the redundancy in the correlated deck simply by eliminating the orthogonal tasks. Without the orthogonal set of stimuli, all decks of cards seen by $S$ contained just two different stimuli. This fact was expected to increase the likelihood that $S$ would note the correlation between the stimulus dimensions.

The implicit condition included four tasks: sort by dimension $\mathrm{A}$ when $\mathrm{A}$ alone varies, sort by $B$ when $B$ alone varies, sort by $A$ when $A$ and $B$ are correlated, and sort by $\mathrm{B}$ when $\mathrm{A}$ and $\mathrm{B}$ are correlated.

Explicit instructions. In a second condition, the instructions were more explicit: $S$ was told at the beginning of the experiment that on some trials the two dimensions would be perfectly correlated, that on those trials $S$ would be told to "sort by both," and that $S$ was then free to sort into two piles by any means he preferred, since there were still only two different stimuli in the deck.

In the explicit condition, therefore, only three tasks were included: sort by $A$ when $A$ alone varies, sort by $B$ when $B$ alone varies, and sort by "both" when A and B are correlated.

\section{Order of Presentation}

For each $S$, test trials were preceded by a block of 12 practice trials. For the test trials one Latin square was formed with four Ss, four tasks, and four orders for the implicit condition, and another with three Ss, three tasks, and three orders for the explicit condition. The appropriate Latin square determined the order of the first four trials in the implicit condition and the first three trials in the explicit condition. The starting order was then reversed for the next block of trials, reversed again, and so on, until a total of 24 test trials was reached. The same 4 by 4 Latin square was used for each of three subgroups of four Ss in the implicit condition, and the same 3 by 3 Latin square for each of four subgroups of three $S s$ in the explicit condition.

\section{Statistical Analyses}

The time taken to sort a deck of 32 cards into two piles was the primary performance measure. For each condition in both experiments a complete-three-way analysis of variance was carried out for Ss by Trials by Tasks. While time scores decreased significantly across trials, this factor did not interact with tasks and will not be further discussed. All statements of significance are at the .01 level unless otherwise noted.

\section{Errors}

As in the Garner and Felfoldy (1970) experiments errors were few, averaging .24 per deck. Rank correlation of times and errors across all experiments, conditions, and tasks was +.75 . Thus, there is little information to be gained from error analysis beyond that provided by the time data.

\section{RESULTS AND DISCUSSION}

Experiment 1: Size of Circle and Angle of Diameter

Stimuli. The first pair of dimensions studied were those used by Garner and Felfoldy (1970) which had the greatest difference in discriminability between the two dimensions and which thus are most apt to produce SSP. The stimulus was a black circle centered on the card, with a diameter drawn through it. The size of the circle was $7 / 8$ in. or 1 in. in diam when size was an operative dimension, and $15 / 16 \mathrm{in}$. in diam when not operative. The angle of the diameter was 50 or $65 \mathrm{deg}$ clockwise from the vertical when operative, and $57.5 \mathrm{deg}$ when not operative. When the dimensions were correlated, the larger angle was associated with the smaller size. Thus the stimuli used were the same as those of the earlier experiment, except that the orthogonal combination of dimensions was not included in the present experiment.

Results: Implicit condition. The mean sorting times for each $\mathrm{S}$ on each of the four tasks are shown in Table 1. Size,was sorted significantly faster than angle when used alone, as expected. (All Ss showed this result.) Sorting by angle when angle was correlated with size was significantly faster than sorting by angle when used alone, but did not reach the speed of sorting by size alone.

Thus, there is evidence that merely eliminating the orthogonal tasks in the card-sorting experiment leads to at least partial use of the SSP strategy. Four of the
Table I

Size of Circle and Angle of Diameter: Implicit Condition. Mean Sorting Times in Seconds for Each of the Four Tasks

\begin{tabular}{|c|c|c|c|c|}
\hline \multirow[b]{2}{*}{$S$} & \multicolumn{4}{|c|}{ Tasks } \\
\hline & $\begin{array}{l}\text { Angle } \\
\text { Alone }\end{array}$ & $\begin{array}{l}\text { Size } \\
\text { Alone }\end{array}$ & $\begin{array}{c}\text { Angle } \\
\text { Corre- } \\
\text { lated }\end{array}$ & $\begin{array}{c}\text { Size } \\
\text { Corre- } \\
\text { lated }\end{array}$ \\
\hline 1 & 52.90 & 25.33 & 35.13 & 29.17 \\
\hline 2 & 27.03 & 18.40 & 21.57 & 19.77 \\
\hline 3 & 22.23 & 14.93 & 21.53 & 15.47 \\
\hline 4 & 17.23 & 15.13 & 15.53 & 13.87 \\
\hline 5 & 26.10 & 19.40 & 21.50 & 19.27 \\
\hline 6 & 23.90 & 19.50 & 22.40 & 18.53 \\
\hline 7 & 19.80 & 16.47 & 17.50 & 15.70 \\
\hline 8 & 25.70 & 17.40 & 23.43 & 18.07 \\
\hline 9 & 20.30 & 16.73 & 16.33 & 15.97 \\
\hline 10 & 21.03 & 15.20 & 14.67 & 14.50 \\
\hline 11 & 23.40 & 17.83 & 20.00 & 17.17 \\
\hline 12 & 18.90 & 17.40 & 18.00 & 18.03 \\
\hline Mean & 24.88 & 17.81 & 20.63 & 17.96 \\
\hline
\end{tabular}

12 Ss improved their speed of sorting by angle with the addition of the correlated dimension of size down to nearly the same level as if sorting by size alone (the result expected with SSP). The other eight Ss all showed improvement in sorting speed, but to some level intermediate between that obtained with angle alone and size alone. This intermediate performance was not due to Ss learning the SSP strategy over successive trials, since the appropriate interaction terms did not approach significance. Rather, it appears that they were using some compromise strategy: They tried to sort according to E's instructions. but were unable to avoid on every trial the use of the more discriminable correlated dimension.

Results: Explicit condition. The mean sorting times for each $\mathrm{S}$ on each of the three tasks are shown in Table 2. Size when used alone was sorted significantly faster than angle when used alone, as in the implicit condition. Sorting on the correlated task was significantly faster than

Table 2

Size of Circle and Angle of Diameter: Explicit Condition, Mean Sorting Times in Seconds

\begin{tabular}{|c|c|c|c|}
\hline \multirow[b]{2}{*}{$S$} & \multicolumn{3}{|c|}{ Tasks } \\
\hline & $\begin{array}{l}\text { Angle } \\
\text { Alone }\end{array}$ & $\begin{array}{c}\text { Size } \\
\text { Alone }\end{array}$ & $\begin{array}{l}\text { Corre- } \\
\text { lated }\end{array}$ \\
\hline 1 & 16.42 & 13.38 & 12.80 \\
\hline 2 & 16.50 & 13.42 & 12.92 \\
\hline 3 & 30.32 & 15.62 & 15.08 \\
\hline 4 & 30.28 & 19.28 & 19.85 \\
\hline 5 & 26.68 & 21.28 & 24.22 \\
\hline 6 & 23.92 & 19.82 & 19.72 \\
\hline 7 & 18.48 & 13.70 & 14.22 \\
\hline 8 & 20.70 & 14.38 & 14.38 \\
\hline 9 & 19.95 & 15.28 & 15.10 \\
\hline 10 & 15.35 & 12.70 & 12.90 \\
\hline 11 & 17.20 & 15.28 & 15.90 \\
\hline 12 & 22.62 & 14.28 & 12.90 \\
\hline Mean & 21.53 & 15.70 & 15.83 \\
\hline
\end{tabular}


Table 3

Value and Chroma in Separate Color Chips: Implicit Condition. Mean Sorting Time in Seconds for Each of the Four Tasks. (The better of the two single dimensions for each $S$ is underlined. The mean of the better of two singles and the mean of the worse of two singles are given below.)

\begin{tabular}{|c|c|c|c|c|}
\hline \multirow[b]{2}{*}{$S$} & \multicolumn{4}{|c|}{ Tasks } \\
\hline & $\begin{array}{l}\text { Value } \\
\text { Alone }\end{array}$ & $\begin{array}{c}\text { Chroma } \\
\text { Alone }\end{array}$ & $\begin{array}{c}\text { Value } \\
\text { Corre- } \\
\text { lated }\end{array}$ & $\begin{array}{l}\text { Chroma } \\
\text { Corre- } \\
\text { lated }\end{array}$ \\
\hline 1 & 16.87 & 15.57 & 15.27 & 15.10 \\
\hline 2 & 13.70 & $\overline{12.43}$ & 13.60 & 13.43 \\
\hline 3 & 13.10 & 12.60 & 12.97 & 13.03 \\
\hline 4 & 18.00 & $\overline{16.53}$ & 16.30 & 16.93 \\
\hline 5 & 13.80 & $\overline{14.23}$ & 14.07 & 14.17 \\
\hline 6 & $\overline{18.63}$ & 18.23 & 18.03 & 18.73 \\
\hline 7 & 15.87 & $\overline{16.33}$ & 16.17 & 15.37 \\
\hline 8 & $\overline{10.80}$ & 11.60 & 10.50 & 11.77 \\
\hline 9 & $\overline{16.10}$ & 16.20 & 14.60 & 15.07 \\
\hline 10 & $\longdiv { 1 5 . 5 0 }$ & 14.20 & 14.13 & 14.33 \\
\hline 11 & 15.77 & $\overline{15.80}$ & 14.70 & 15.77 \\
\hline 12 & $\overline{18.10}$ & 17.93 & 17.97 & 18.50 \\
\hline Mean & 15.52 & 15.14 & 14.86 & 15.18 \\
\hline
\end{tabular}

Note-Better alone equal 14.99. worse alon cqual 15.67

sorting by angle alone and not significantly different from sorting by size alone. Thus the average results clearly showed SSP.

The extent of the use of the SSP strategy is great: Eleven of the $12 \mathrm{Ss}$ showed sorting speeds for the correlated task which were faster or nearly as fast as speeds for size alone. Only $S 5$ failed to show clear evidence of SSP, and this one $S$ sorted at a speed intermediate between that for size and that for angle. Thus the addition of the explicit description of the nature of the correlated task produced almost exclusive use of the SSP strateg. There is no question, then. of the ability of Ss to use this strategy when it is effective to do so and when they are aware of its appropriateness.

\section{Experiment 2: Value and Chroma in Separate Color Chips}

$S S P$ or integration?" As noted above. improvement in performance with correlated dimensions can ocur either because of the use of an SSP strategy or because information from the two dimensions is integrated. Ciamer and Felfoldy (1970) previously argued that the size-angle dimensions were a nonintegral pair; therefore. integration of information is unlikely. However, when two dimensions differ greatly in discriminability, as in Experiment 1, it is almost impossible to detect information integration if it does occur. Any model of the combined discriminability will show only slightly greater discriminability for a pair of unequally discriminable dinmensions than for the better of the two dimensions alone. To illustrate, a Euclidean integration of two dimensions when one is half as discriminable as the other will give a combined discriminability only $11 \%$ greater than that of the better dimension alone.

Thus our use of a condition which maximized the possibility of Ss' using an SSP strategy also minimized the possibility of the data's clearly showing information integration. Equal discriminability of two dimensions will maximize the effectiveness of integrating information, while at the same time minimizing the effectiveness of SSP strategies. This condition (with otherwise clearly nonintegral dimensions) was met in the Garner and Felfoldy experiments with the use of the Munsell dimensions of value and chroma varving in two separate color chips.

If the paradigm of Experiment 1 is applied to this pair of dimensions, with its balance shifted toward integration as opposed to SSP. we may be able to determine more clearly which mode of processing is being facilitated by implicit and explicit instructions concerning redundancy.

Stimuli. The dimensions used were the Munsell dimensions of value and chroma. varying in two separate glossy $5 \mathrm{R}$ color chips. Chips were $0.75 \mathrm{in}$. square and separated by 19/32 in. When value alone varied, Value 5 , chroma 4 was contrasted with Value 6, Chrona 4 on the upper chip. while the lower chip was held constant at Value 5. Chroma (1. When chroma alone varied, the upper chip was held constant at Value 5. Chroma 6, while the bottom chip assumed levels of Value 6 , Chroma 4 or Value 6. Chroma 6 . When the dimensions were correlated, one stimulus card had Value 5. Chroma 4 on iop with Value 6 Chromat on the boltom. while the other stimulus card had Value 6. Chroma 4 on (up) with Value o. Chromat on the buttom.

Results: Implicit condition. The mean sorting times for each $S$ on each of the four tasks are shown in Table 3. Chroma was not sorted significantly faster than value when used alone. Sorting by value when value wats correlated with chroma was significantly lister than sorting by value when alone, but was not significantly laster than sorting by chromat alone or by cliroma when correlated with value.

At lirst glance, the significant redundancy gain on the value dimension would appear to indicate that a small amoun of integration had taken place with these nonintegral dimensions. Yet the strongest test for information integration, as opposed to SSP. in these experiments is that $\mathrm{Ss}$ sort faster in the conclated task than in the fiaster of the two single tasks. We have already seen in Experiment 1 that evidence for SSP has been obtained under
Table 4

Value and Clnroma in Separate Color Chips: Explicit Condition. Mean Sorting Time in Seconds for Each of the Three Tasks. (The better of the two single dimensions for each $S$ is underlined. The mean of the better of two singles and the mean of the worse of two singles are given below.)

\begin{tabular}{|c|c|c|c|}
\hline $\mathrm{S}$ & $\begin{array}{l}\text { Value } \\
\text { Alone }\end{array}$ & $\begin{array}{c}\text { Chroma } \\
\text { Alone }\end{array}$ & $\begin{array}{c}\text { Corre- } \\
\text { lated }\end{array}$ \\
\hline I & 13.52 & 14.18 & 13.38 \\
\hline 2 & 12.65 & 11.30 & 11.15 \\
\hline 3 & 14.98 & $\overline{15.65}$ & 14.70 \\
\hline 4 & $\overline{16.50}$ & 16.02 & 16.52 \\
\hline 5 & 13.00 & $\overline{13.32}$ & 13.00 \\
\hline 6 & 13.50 & 13.88 & 13.80 \\
\hline 7 & $\overline{13.20}$ & 12.90 & 13.42 \\
\hline 8 & 19.80 & 18.78 & 19.48 \\
\hline 9 & 14.45 & $\overline{14.32}$ & 14.00 \\
\hline 10 & 13.25 & $\overline{14.42}$ & 14.28 \\
\hline 11 & 17.28 & 16.60 & 16.60 \\
\hline 12 & 16.92 & 16.55 & 16.28 \\
\hline Mean & 14.92 & 14.83 & 14.72 \\
\hline
\end{tabular}

Vote-Bctter alone equal 14.56, worse alone equal 1.5 .19 .

the conditions of the present experiments, so that the most parsimonious assumption is that a gain to the level of the better of the two dimensions alone is the result of SSP. A gain significantly beyond that level is an unambiguous indication that integration of information has occurred.

In Table 3. the better of the two single dimensions for each $\mathrm{S}$ is underlined, and the nuean of these underlined scores is indicated below. Neither mean for the correlated lasks is significantly lower than this level. Furthermore, only seven Ss sorted faster than their best single dimension when sorting by value with correlated dimensions, and only 3.5 Ss sorted faster than their best single dimension when sorting by chroma with correlated dimensions. Thus the data fail to show any evidence for integration.

Neither of the two necessary conditions for effective SSP was satisfied: Both the main effect of dimensions and the interaction of $\mathrm{Ss}$ by Dimensions failed to reach significance. One would thus expect scant evidence for SSP in this experiment. The argument for SSP is necessarily a double logical onc: that Ss perform significantly better on the correlated tasks than on the worse of the single dimensions. but not significantly better than the lower of the single dimensions. The latter requirement has already been shown to have beell satisfied. The mean scores for both valuc and chroma when correlated are lower $(p<.01$ and $p<.05$, respectively) than the mean of the worse of the two singles, as seen in Table 3 . In terms of individual Ss, all 12 sorted faster on the value-currelated task and 9 Ss sorted faster on the chroma-corrclated task than on the worse of the two singles. Thus there is 
some evidence for a small amount of SSP in this experiment and no evidence for integrality.

Results: Explicit condition. Table 4 shows the mean sorting times for each $S$ on each of the three tasks. No significant differences between the means for the three tasks were found. The means of both the better of the two singles and the worse of the two singles are shown at the bottom of the table. The mean for the correlated task is not significantly different from the mean of the better of the two singles, and only six Ss showed improvement on an individual basis.

Since neither the main effect due to dimensions nor the interaction of Ss by Dimensions was significant, the opportunity for SSP was minimal. Nevertheless, the mean for the correlated task is significantly lower $(p<.05)$ than the worse of the two single-dimension tasks, with 10 of the Ss showing better performance. These results once again show no evidence for information integration, and some weak evidence for a small amount of SSP with these stimuli.

\section{CONCLUSIONS}

The principal conclusion to be drawn from these data is that Ss use SSP in a speeded classification task when two stimulus dimensions are correlated and when they are made aware of the correlation. Implicitly instructing Ss of the existence of the correlated dimension, by using only tasks having just two alternative stimuli, produces SSP by at least some Ss on some trials. The addition of an explicit description of the correlated task produces nearly total use of the SSP strategy.

The surprising lack of evidence for SSP in the Garner and Felfoldy (1970) paper appears to have been due to the specific conditions used and the instructions given to $S$, rather than to the nature of the card-sorting task itself. Ss do use sorting strategies which optimize their performance, once they become aware of their availability and effectiveness.

The two pairs of dimensions used in the present experiments were chosen from those found by Garner and Felfoldy (1970) to be nonintegral; they produced no gain when combined redundantly and no interference when combined orthogonally in the card-sorting task. In the present experiments conditions were manipulated so as to emphasize the effectiveness of an SSP sorting strategy, and no redundancy gains were found which could not be accounted for adequately by the use of such a strategy. Thus the results were consistent with the concept of stimulus integrality in general, and with the conclusion that the dimensions of value and chroma in separate chips and of size of circle and angle of diameter are properly classed as nonintegral.

\section{REFERENCES}

GARNER, W. R, \& FELFOLDY, G. L. Integrality of stimulus dimensions in various types of information processing. Cognitive Psychology, 1970, 1, 225-241.

HYMAN, R., \& WELL, A. Perceptual separability and spatial models. Perception \& Psychophysics, 1968, 3, 161-165.

SHEPARD, R. N. Attention and the metric structure of the stimulus space. Journal of Mathematical Psychology, 1964, I, 54-87.

(Accepted for publication July 27, 1970.) 